\section{P-31 A NEW HOSPICE PROJECT ROLE: CLINICAL COMMUNITY ENGAGEMENT LEAD}

Caroline Mundy. St Peter's Hospice, Bristol, UK

\subsection{6/bmjspcare-2019-HUKNC.55}

Background People living and dying with non-malignant conditions, and those from minority communities face inequalities in both access to, and provision of, end of life care (Care Quality Commission, 2016; Tackling Inequalities in End of Life Care for Minority Groups VCSE Health and Wellbeing Alliance Project Group, 2018; Our commitment to you for end of life care. The government response to the review of choice in end of life care. Department of Health 2016). The length of time hospice care is given is shorter for those with non-malignant conditions (Allsop, Ziegler, Mulvey, Russell et al., 2018). This new two-year project role started in April 2019.

Aims To improve access and equity of access to hospice services; increase understanding of the diverse needs of our community; be recognised as a valued community-based service and effectively communicate the hospice role to health professionals and the wider public; seek out and effectively manage partnerships.

Methods First 12 months: April-August 2019; define and scope current situation; review current patient and local population data/demographics. Literature review. Map current partnerships and pathways. Survey staff to identify self-reported confidence and competence in providing care and support identify training needs. Map existing available services in acute trusts and the community. Engage with external health and social care organisations. August 2019-March 2020: gap needs analysis of current service provision. Root cause analysis to identify barriers that minority groups might face accessing end of life care services. Continue with engagement with local partners.

Results We anticipate a greater understanding of the hospice's role in meeting the palliative care needs of patients with nonmalignant conditions. We seek to use these data and increased knowledge to assess and explore potential changes in service model delivery in response to identified unmet need. We aim to enable staff to provide personalised high quality end of life care regardless of diagnosis, age, ethnic background, sexual orientation, gender identification, disability or social circumstances through the development of training. We anticipate stronger partnership working to provide high quality end of life care.

Conclusions A proactive approach by the hospice in reviewing services and improving understanding/needs. An evaluation process of the role will occur.

\section{P-32 INCURABLE CONDITIONS: UNDERSTANDING THE VIEWS AND EXPERIENCES OF THE SOUTH ASIAN COMMUNITY}

Karan Jutlla, Marianne Grant. Compton Care, Wolverhampton, UK

\subsection{6/bmjspcare-2019-HUKNC.56}

Recent population projections identify that ageing trends are expected to continue. By 2026 it is estimated that there will be over 1.3 million people from Black, Asian and Minority Ethnic (BAME) groups aged 65 or older in the UK, many of whom may experience life limiting illnesses that could benefit from care. Latest figures from Wolverhampton City Council (2017) suggest a population of 259,926 (5,000 increase since the 2011 census) with 35.5 per cent of residents being from a BAME community, and yet service uptake from such communities has been considerably low.

Funded by the Big Lottery Community Fund, this project aimed to address the inequalities which exist around support for the South Asian Community in Wolverhampton. Through a series of community engagement activities and focus groups, we captured the views, thoughts and experiences of the South Asian community in Wolverhampton when living with or caring for someone with a complex, advanced or incurable condition.

This poster highlights the main findings from this project with recommendations for policy and practice in palliative care services across the UK.

\section{P-33 TALKING DEATH AND BEREAVEMENT WITH A SOUTH ASIAN WOMEN'S GROUP IN WALSALL}

${ }^{1}$ Alpa Thakar, ${ }^{2}$ Margaret Brown. ${ }^{1}$ St Giles Hospice, Walsall, West Midlands; ${ }^{2}$ St Giles Hospice, Lichfield, West Midlands

\subsection{6/bmjspcare-2019-HUKNC.57}

Background St Giles Hospice has been delivering hospice care in Walsall since 2011. Data showed that patients from certain groups within the local community have not accessed hospice care.

Our Community Engagement Officer for Walsall was introduced to a group of women, mainly of South Asian origin (India, Pakistan and Bangladesh), who met on a weekly basis at a local community hub. The group were curious to know more about the work of the hospice and the role of community engagement. Taster sessions were facilitated which identified that the women had little opportunity to discuss matters around death, dying and bereavement within their own families or communities.

Aim To facilitate monthly sessions where women from different cultural backgrounds could openly discuss matters around death, dying and bereavement and be more informed about hospices and end of life care.

Method In partnership with the group we then undertook monthly sessions incorporating a range of activities including memory jars, Bollywood film clips, emotional first aid kits etc. which were used as 'tools' to facilitate conversations - coupled with 'having fun' which was a 'must' for the women.

Results Deep and insightful conversations took place. Women were able to share personal stories of death, dying and bereavement in a safe and supportive environment, including discussing what 'good' end of life care means to them. As an organisation we have also benefited and have developed our insight and understanding, informing our approach to practice.

Conclusion Through adapting our conventional approach these sessions have demonstrated the value of using a conversational community engagement approach when raising awareness of hospices and end of life support. Women who previously hadn't had the opportunity to express and share their thoughts and feelings about this important subject within their families or communities have been given a voice. 